Acta bot. bras. 1(2):95-102 (1988) supl.

\title{
LEVANTAMENTO DA FLORA VASCULAR RUPESTRE DO MORRO SAPUCAIA E MORRO DO CABRITO, RIO GRANDE DO SUL
}

\author{
Irene Fernandes $(1,2)$ \\ Luis Rios de Moura Baptista (2)
}

\begin{abstract}
RESUMO - Apresentam-se os resultados preliminares do levantamento da flora vascular que ocorre em lugares rochosos do Morro Sapucaia e Morro do Cabrito, Rio Grande do Sul, Brasil. O trabalho em andamento inclui observaçōes sobre o hábito, habitat, fenologia e distribuiçāo geográfica das espécies, e uma descrição sucinta da vegetação. Foram encontradas, até novembro de 1986 , 71 espécies de plantas vasculares, sendo 28 de pteridófitas e 43 de angiospermas.
\end{abstract}

ABSTRACT - Preliminary results of the vascular flora survey in rocky places at two mounts in Rio Grande do Sul are presented. The research, which is going on, includes observation on habit, habitat, phenology and geographical distribution of the species as well as a brief description of vegetation. Twenty-eight fern and 43 angiosperm species have been found until November 1986.

Key-words: vascular rock flora, sandstone monadnock, Rio Grande do Sul.

\section{Introdução}

Vegetação rupestre ou rupícola é aquela que prospera em substratos de natureza rochosa, desprovidos ou quase de terra vegetal.

Além dos problemas de escassez de solo, as comunidades rupícolas podem enfrentar variações térmicas muito acentuadas e disponibilidades hídricas irregulares. Geralmente trata-se de plantas especializadas em colonizar esse tipo de lugar (Folch i Ghillèn 1981).

O presente trabalho tem por objetivo realizar o levantamento da flora vascular que ocorre em lugares rochosos do Morro Sapucaia e Morro do Cabrito, Rio Grande do Sul, incluindo dados sobre o hábito, habitat, fenologia e distribuição geográfica das espécies encontradas e ainda uma descrição sucinta da vegetação, salientando as espécies importantes.

\section{Locais de Estudo}

Situação Geográfica. As áreas em estudo estão situadas na região fisiográfica denominada Depressão Central, no Rio Grande do Sul, Brasil (Rambo 1956).

O Morro Sapucaia está localizado na Microrregião de Porto Alegre, município de Sapucaia do Sul, na latitude de $29^{\circ} 50^{\prime}$ S e longitude de $51^{\circ} 06^{\prime} \mathrm{W}$ Gr. Sua altitude é de $290 \mathrm{~m}$.

O Morro do Cabrito está localizado na Microrregião Colonial da Encosta da Serra Geral, município de Montenegro, na latitude de $29^{\circ} 38^{\prime} \mathrm{S}$ e longitude de $51^{\circ} 39^{\prime} \mathrm{W}$ Gr. Tem uma altitude de, aproximadamente, $270 \mathrm{~m}$.

(1) Dep. de Botânica, UFRGS - Av. Paulo Gama s/nº, 90040, Porto Alegre, RS, Brasil.

(2) Bolsista do CNPq 
Clima. Os dados ćlimáticos para a região da Depressão Central, onde se localizam estes morros, baseados em Machado (1950), são os seguintes: temperatura média anual de $19,4^{\circ} \mathrm{C}$; precipitação normal anual de $1300-1800 \mathrm{~mm}$; geada abundante; direção predominante dos ventos SE-NE; insolação normal anual de 2258 horas; umidade relativa do ar, valores normais, $73-85 \%$. É grande a formação de nevoeiros, notadamente no centro e leste da região.

O clima enquadra-se no tipo subtropical, Cfa, da classificação de Köppen, que é o tipo predominante no Rio Grande do Sul.

Geomorfologia. Os locais em estudo são formações típicas da Depressão Central do Rio Grande do Sul. São morros testemunhos, resíduos da antiga área ocupada pelo planaltỏ cujas escarpas recuam atacadas pela erosão (Rambo 1956, Penteado 1983). Os dois morros são tabuleiros areníticos com o topo quase plano e as escarpas abruptas (Figs. 1 e 2).

Uma das propriedades geomorfológicas do arenito é ser uma rocha de fácil permeabilidade. A água da chuva se infiltra com rapidez ao longo da própria superfície ou das fraturas (Penteado 1983). Estas águas de infiltração vão sendo liberadas aos poucos e mantêm úmidas muitas paredes ou escorrem ao longo delas, permanentemente. Além disso, o arenito é uma rocha friável que, atacada pela erosão, apresenta-se fendilhada, com ressaltos, covas, plataformas, chaminés, etc. Assim, ao longo dos paredões encontramos uma infinidade de diferentes tipos de habitat que vão desde paredes expostas ao sol pleno, ventos e geadas, até locais abrigados, permanentemente sombrios e úmidos.

\section{Material e Métodos}

Escolha das áreas de estudo. Considerou-se a importância fisionômica e científica dos morros testemunhos por seu relevo e vegetação característicos. Rambo (1956) referu-se ao Morro Sapucaia: "Este Morro é um museu natural contendo todos os elementos essenciais da geologia, vegetação e formas individuais do arenito da Depressão Central..."

Há urgência de um estudo mais aprofundado destes locais porque muitos dos morros da Depressão Central se localizam na região de maior densidade populacional do Estado e têm sua fisionomia natural ameaçada por danos decorrentes da invasão urbana (Metroplan 1978).

Foram escolhidos morros com ocorrência de vegetação em locais rochosos em todas as exposições.

O Morro do Cabrito tem suas escarpas tão íngremes que tornam a subida impossível sem equipamento especial. Por isso supõe-se que sua vegetação esteja preservada, até certo ponto, da depredação pelo homem, constituindo-se assim em excelente local para o estudo da vegetação rupestre.

Delimitação. Decidiu-se coletar todas as plantas vasculares que ocorrem diretamente sobre a rocha ou em fendas na rocha, excluindo-se a vegetação sobre rochas que ocorrem no meio da mata que cerca os paredões rochosos dos morros, por necessidade de reduzir-se a área de coleta e observação.

Amostragem. A partir de dezembro de 1985 estão sendo realizadas excursões mensais para coleta de plantas e levantamento de dados e continuarão até setembro de 1987.

Está sendo realizada uma amostragem com o objetivo de abranger a maior área possível porque verificou-se que a variação de fatores como a exposição, inclinação, conformação do substrato e outros, origina uma sucessão de muitos tipos diferentes de habitat ocupados por muitas espécies distintas.

Para atingir locais de acesso mais difícil, são utilizadas técnicas e material de escalada. 


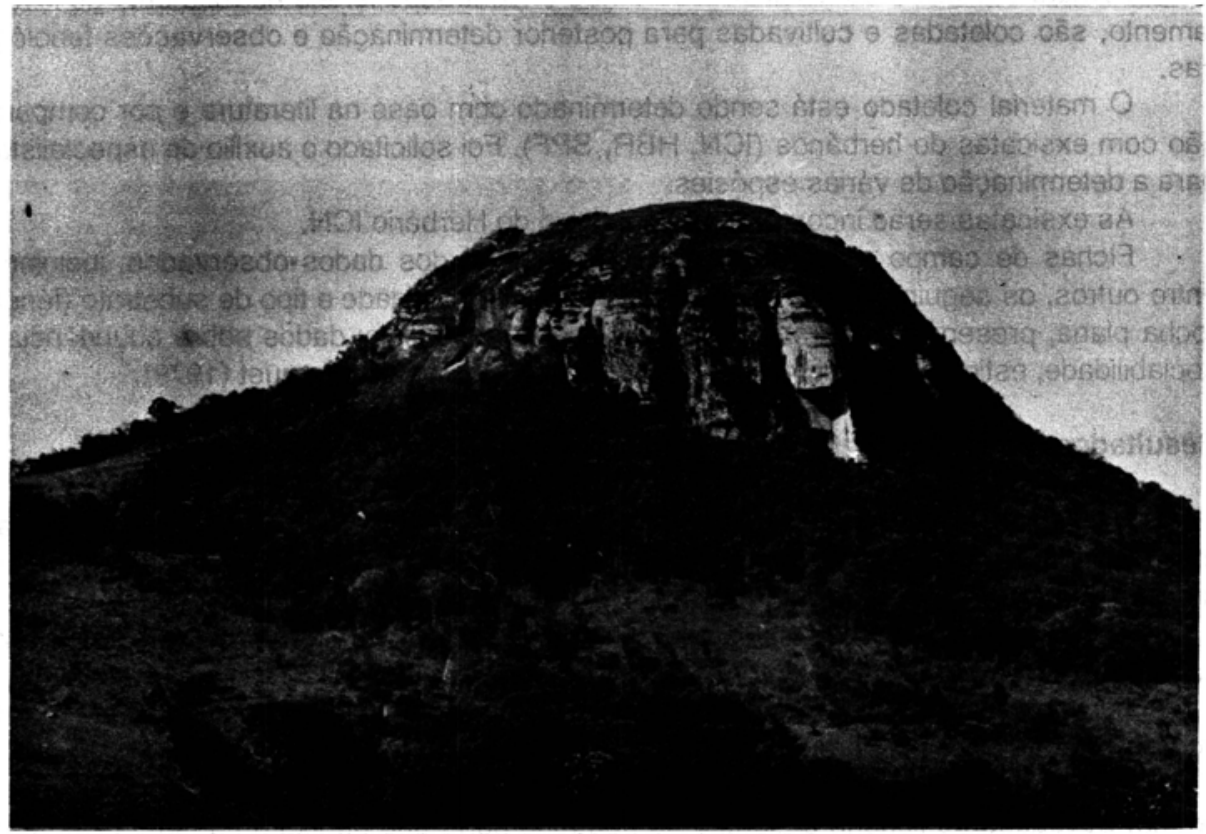

Fig.1 - Morro Sapucaia

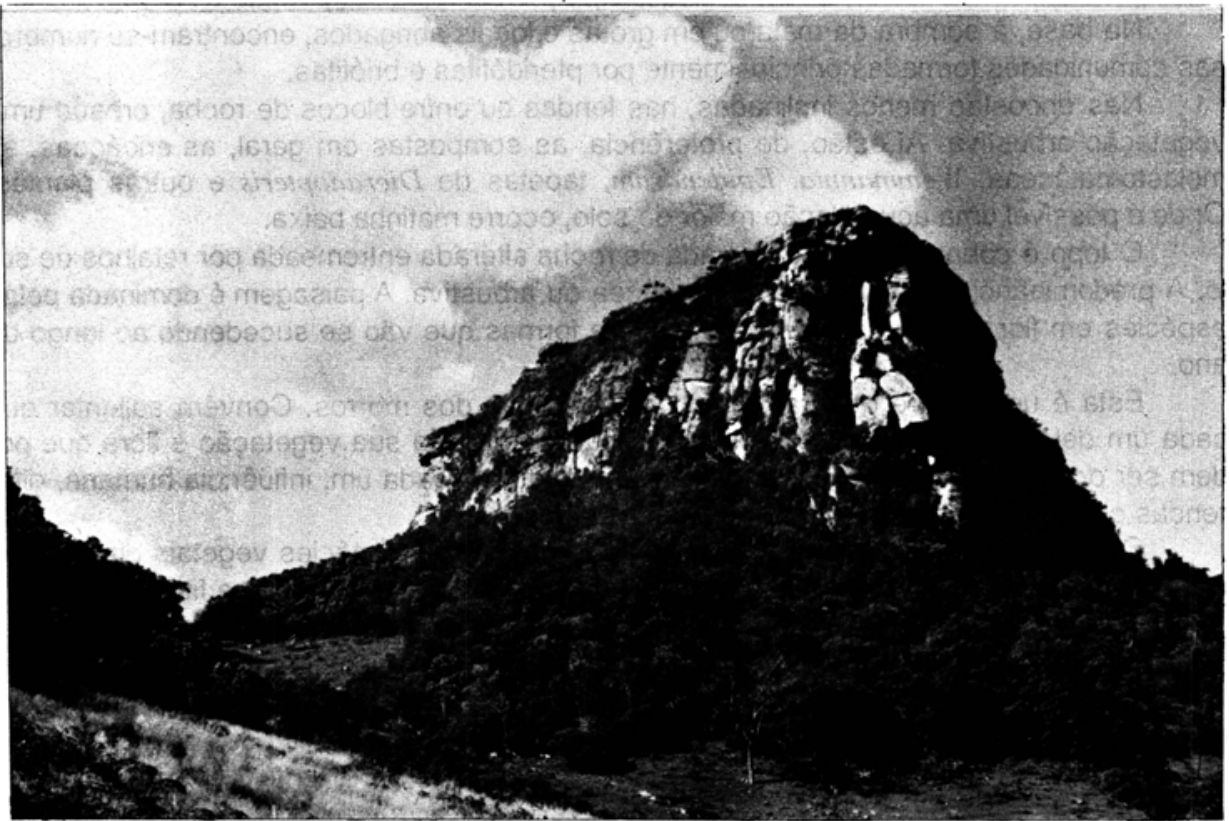

Fig.2 - Morro do Cabrito 
Plantas em locais de diff́cil acesso e não encontradas férteis no momento do levantamento, são coletadas e cultivadas para posterior determinação e observações fenológicas.

O material coletado está sendo determinado com base na literatura e por comparação com exsicatas de herbários (ICN, HBR, SPF). Foi solicitado o auxilio de especialistas para a determinação de várias espécies.

As exsicatas serão incorporadas ao acervo do Herbário ICN.

Fichas de campo são utilizadas para anotação dos dados observados, incluindo, entre outros, os seguintes itens: exposição, inclinação, umidade e tipo de substrato (fenda, rocha plana, presença de serrapilheira, etc.); hábito; fenologia; dados sobre abundância e sociabilidade, estimativa visual baseada nas escalas de Braun-Blanquet (1979).

\section{Resultados e Conclusão}

Na Tabela 1 está sendo apresentada uma lista preliminar das espécies encontradas até novembro de 1986, constituída de 71 espécies de plantas vasculares, sendo 28 de pteridófitas e 43 de angiospermas.

As pteridófitas estão distribuídas em 12 famnlias e 16 gêneros. $O$ sistema de classificação adotado é o de Tryon e Tryon (1982).

As angiospermas estão distribuídas em 16 famnias e 32 gêneros, clașificadas de acordo com o sistema de Cronquist (1981).

A vegetação dos morros. Cercados de mata, os paredões dos morros se elevam a prumo, povoados de líquens e plantas de pequeno porte que se fixam nas irregularidades da superfície.

No alto dos paredões, predominam as bromeliáceas (gêneros Tillandsia, Dyckia e Vriesea), orquidáceas (principalmete Cattleya e Bifrenaria), cactáceas (Rhipsalis), gramíneas (principalmente Axonopus e Andropogon) e pteridófitas (Polypodium e Microgramma).

$\mathrm{Na}$ base, à sombra da mata ou em grotas e locais abrigados, encontram-se numerosas comunidades formadas principalmente por pteridófitas e briófitas.

Nas encostas menos inclinadas, nas fendas ou entre blocos de rocha, cresce uma vegetação arbustiva. Aí estão, de preferência, as compostas em geral, as ericáceas, as melastomatáceas, Weinmannia, Epidendrum, tapetes de Dicranopteris e outras plantas. Onde é possível uma acumulação maior de solo, ocorre matinha baixa.

O topo é coberto por uma camada de rocha alterada entremeada por retalhos de solo. A predominância é de vegetação herbácea ou arbustiva. A paisagem é dominada pelas espécies em flor com suas diferentes cores e formas que vão se sucedendo ao longo do ano.

Esta é uma breve generalização da vegetação dos morros. Convém salientar que cada um deles apresenta alguns aspectos particulares na sua vegetação e flora que podem ser ocasionados pelas diferenças na topografia de cada um, influência humana, diferenças climáticas das áreas onde estão localizados, etc.

Os dados apresentados mostram grande riqueza de espécies vegetais distribuídas em diferentes çomunidades, as quais variam na composição florística e na fisionomia.

Com base nas observações de campo, conclui-se que esta riqueza de espécies e formas fica explicada pela grande variedade de habitats que estes morros apresentam.

Considera-se importante a extensão destes estudos a outros morros, permitindo um maior conhecimento de sua flora característica. 


\section{Agradecimentos}

A S. Boechat, N.I. Matzenbacher, M.E. Sobral, J.L. Waechter (UFRGS - Porto Alegre), A. Chautems (Conservatoire et Jardin Botaniques - Genebra) e P.G. Windisch (UNESP - São José do Rio Preto), agradecemos pelo auxilio na determinação de algumas espécies.

\section{Referências Bibliográficas}

BRAUN-BLANQUET, J. 1979. Fitosociologia: bases para el estudio de las comunidades vegetales. H. Blume, Madrid.

CRONQUIST, A. 1981. An integrated system of classification of flowering plants. Columbia University, New York.

FOLCH i GUILLÈN, R. 1981. La vegetació dels Paísos Catalans. Ketres, Barcelona.

MACHADO, F.P. 1950. Contribuição ao estudo do clima do Rio Grande do Sul. IBGE, Rio de Janeiro.

METROPLAN-FUNDAÇĀO METROPOLITANA DE PLANEJAMENTO. 1978. Estudo de destinação do uso do solo rural na Região Metropolitana de Porto Alegre - ano 1976. Porto Alegre.

PENTEADO, M.M. 1983. Fundamentos de geomorfologia. 3.ed. IBGE, Rio de Janeiro.

RAMBO, B. 1956. A fisionomia do Rio Grande do Sul. 2.ed. Selbach, Porto Alegre.

TRYON, R.M.\& TRYON, A.F. 1982. Ferns and Allied Plants. Springer-Verlag, New York. 
TABELA 1 - Lista das espécies de plantas vasculares rupestres encontradas nas áreas em estudo.

Siglas: MS - Morro Sapucaia; MC - Morro do Cabrito

a - Pteridófitas

\section{Famnias/espécies}

Aspleniaceae

Asplenium serra Langsd. \& Fisch.

Blechnaceae

Blechnum exiguum Dutra

B. meridense (KI.) Mett.

$B$. occidentale $\mathrm{L}$.

$B$. serrulatum Rich.

B. unilaterale $\mathrm{Sw}$.

Cyatheaceae

Trichipteris atrovirens (Langsd. e Fisch.) Tryon

Dennstaedtiaceae

Histiopteris incisa (Thunb.) J. Sm.

Dryopteridaceae

Elaphoglossum"sp.

E. burchellii (Baker) C. Chr.

E. macrophyllum (Mett.) Chr.

Rumohra adiantiformis (Forst.) Ching

Gleicheniaceae

Dicranopteris flexuosa (Schrad.) Underw.

Hymenophyllaceae

Hymenophyllum hirsutum (L.) Sw.

Trichomanes angustatum Carm.

$T$. pilosum Raddi

Lophosoriaceae

Lophosoria quadripinnata (Gmel.) C. Chr.

Osmundaceae

Osmunda cinnamomea $\mathrm{L}$.

Polypodiaceae

Microgramma squamulosa (Kaulf.) Sota

M. vacciniifolia (Langsd. \& Fisch.) Copel.

Polypodium aureum $\mathrm{L}$.

$P$. catharinae Langsd. \& Fisch.

P. lepidopteris (Langsd. \& Fisch.) Kze.
MS

MC

$x$

$x$

$x$

$x$

$x$

$x$

$x$

$x$

$x$

$x$

$x$

$x$

$x$

$x$

$x$

$\mathrm{X}$

$x$

x

x

$x$

$x$

$x$

X

X

X

$x$

$x$

$\mathrm{X}$

$x$

$x$

$x$

$x$

x


b - Angiospermas

\section{Araceae}

Anthurium scandens (Aubl.) Engl.

Asteraceae

Achyrocline satureioides DC.

Baccharis mesoneura DC.

B. tridentata Vahl

Carelia ramboi Cabr.

Senecio heterotrichus DC.

Symphyopappus reticulatus Baker

$\begin{array}{ll}X & X \\ X & X \\ X & X \\ X & X \\ X & X\end{array}$

Pteridaceae

Doryopteris triphylla (Lam.) Christ

Eriosorus myriophyllus (Sw.) Copel.

Pityrogramma calomelanos (L.) Link

$\begin{array}{ll}x & x \\ x & x \\ & x\end{array}$

Vittariaceae

Vittaria graminifolia Kaulf.

$V$. lineata (L.) Sm.

$x$
$x$

$x$

Bromeliaceae

Aechmea recurvata L.B. Smith

Dyckia cf. alba Winkler

$x$

D. maritima Baker

Tillandsia sp. 1

T.sp. 2 .

T. aeranthos (Loiseleur) L.B. Smith

T. geminiflora Brongn.

T. lorentziana Griseb.

T. usneoides (L.) L.

Vriesia friburgensis Mez

$x$

$x$
$x$

Bignoniaceae

Pyrostegia venusta (Ker-Gawl.) Miers.

$x$

$x$

Cactaceae

Rhipsalis baccifera (J. Miller) Stearn.

$R$. cruciformis Castellanos

$\begin{array}{ll}x & x \\ x & x\end{array}$

$x \quad x$

Caryophyllaceae

Paronichia chilensis DC.

$x$

Cunoniaceae

Weinmannia paulliniifolia Pohl ex Serıge

$x$

$x$ 
cont.

\section{Cyperaceae}

Carex sp.

$\mathrm{X} \quad \mathrm{X}$

Ericaceae

Gaylussacia brasiliensis (Spr.) Meissner

Leucothoe nummularia (Cham. et Schl.) DC.

$\begin{array}{ll}x & x \\ x & x\end{array}$

Gesneriaceae

Gesneria macrostachya Lindl.

$\mathrm{X}$

Melastomataceae

Leandra sp.

Miconia sp.

M. sellowiana Naud.

Tibouchina gracilis Cogn.

$T$. herbacea (DC.) Cogn.

$\begin{array}{ll}x & x \\ x & x \\ x & x \\ x & x\end{array}$

Orchidaceae

Bifrenaria harrisoniae (Hook.) Rchb. f.

Cattleya intermedia Graham.

Epidendrum fulgens Brongn.

Pleurothallis glumacea Lindl.

$\begin{array}{ll} & X \\ X & X \\ X & X\end{array}$

Piperaceae

Peperomia sp

$P$. pereskiaefolia (Jacq.) H.B.K.

$\begin{array}{ll}x & x \\ X\end{array}$

Poaceae

Andropogon lateralis Nees

Axonopus sp.

$\begin{array}{cc}X & x \\ X & x \\ & x\end{array}$

Panicum sp.

$\mathrm{X}$

Rubiaceae

Relbunium ericoides (Lam.) K. Schumann

$\mathrm{X} \quad \mathrm{X}$

Scrophulariaceae

Esterhazya splendida Mikan 